\title{
Experimental syphilis vaccines in rabbits I. Differential protection with an adjuvant spectrum
}

\author{
A. M. JONES, J. A. ZEIGLER, AND R. H. JONES \\ Medical Research Institute, Florida Institute of Technology, Melbourne, Florida, U.S.A.
}

\section{Summary}

This study shows that 1 per cent. glutaraldehyde fixation of unwashed Treponema pallidum, Nichols pathogen, preserves the integrity of the outer envelope as well as the ruthenium-red (RR) staining extracellular slime layer. Since other researchers wash their treponemal suspensions before preparing their vaccines, it is likely that the vaccine preparations used in this study contain antigens not tested elsewhere.

In an attempt to eliminate the requirement of large numbers of injections to achieve any protection, the rabbits in this study were injected with glutaraldehyde-fixed $T$. pallidum (GFTP) only once, with or without one of a spectrum of adjuvants, and 5 months later were challenged intradermally or intratesticularly with freshly harvested pathogenic treponemes. Data of time of lesion appearance and severity of lesions reveal a differential protective effect of the various vaccine preparations when compared to the response of non-vaccinated controls. Vaccines which included phytohaemagglutinin (PHA) or alumina $\mathrm{Cgel}$ (ALC) as adjuvants provided some degree of protection, whereas vaccines which contained the adjuvants incomplete Freund's, zymosan, complete Freund's, polyadenylic-polyuridylic acid, or no adjuvant at all produced enhanced infections. The differential protection data indicate that various portions of the immune mechanism are either suppressed or are not stimulated by $T$. pallidum antigens alone, but can be made to respond with a minimum of vaccine injections.

Received for publication September 10, 1975

Address for reprints: Prof. A. M. Jones, Ph.D., Division of Immunology, Medical Research Institute, Florida Institute of Technology, Melbourne, Florida 32901, U.S.A.

\section{Introduction}

To date, published reports have provided little evidence for an effective yet practical syphilis vaccine. Generally, the experimental vaccines have been either non-protective or impractical because of the large number of injections required for protection, for example Metzger, Michalska, Podwinska, and Smogor (1969) 28 injections in 7 weeks and Miller (1973) sixty injections in 37 weeks. Reasons for the limited success of various vaccine preparations are not well understood. However, even the two most successful experimental syphilis vaccines developed possess $T$. pallidum cells initially washed up to three times in saline (Miller, 1973) or stored in saline at $4^{\circ} \mathrm{C}$. for 10 days (Metzger and others, 1969). Metzger and Podwinska (1967) showed that freshly isolated, washed $T$. pallidum undergo a dramatic 4-fold increase in cardiolipin and heat-labile antigenic activity, and a 2-fold increase in heat-stable antigenic activity from 0 to 8 days if the treponemes are kept in phosphate-buffered saline (PBS) at $4^{\circ} \mathrm{C}$. Jones, Nevin, Guest, and Logan (1968) showed by electron microscopy that harvests of unwashed pathogenic $T$. pallidum (Nicholls) cells have an electron dense material on their outer surface, which they indirectly proved aids in the preservation of the integrity of the treponeme. Only 8 per cent. of cells once washed in buffer remained intact after treatment with trypsin, lysozyme, butanol, and complement, compared to 90 per cent. of unwashed cells receiving the same treatment. After washing, the outer envelope was structurally more affected by the trypsin or butanol, whereas lysozyme had a greater effect on the protoplasmic cylinder.

Work by Zeigler, Jones, Jones, and Kubica (1976) clearly demonstrates an extra-cellular slime layer on pathogenic $T$. pallidum using RR staining techniques and electron microscopy. Therefore, it was logical to attempt to 'preserve' these extracellular treponemal components in the procedures used for harvesting treponemes from infected tissues, so as to prevent loss 
of possible protection-inducing antigens. This was the first objective of the present study. Glutaraldehyde was chosen as the fixative because it acts very rapidly with proteins to stabilize their structure by cross-linking them before there is an opportunity for release or leaching of cellular components by the buffer (Glauert, 1975). The retention of the RR staining material was demonstrated in our glutaraldehydefixed preparations of freshly harvested $T$. pallidum.

A further objective of this investigation was to reduce the large number of injections required for protection. The use of adjuvants was an obvious choice, although previous syphilis vaccines using Freund's adjuvant (Magnuson, Halbert, and Rosenau, 1947; Eagle and Fleischman, 1948; Waring and Fleming, 1951 ; Izzat and Yazdi, 1973) or Escherichia coli lipopolysaccharide (Izzat, Knox, Dacres, and Smith, 1971) were generally unsuccessful. Since many different kinds of substances have been shown to stimulate the immune mechanism, a variety of adjuvants other than Freund's was included in this study.

A third objective, that of gaining insight into the mechanism of syphilitic immune reactivity, was attempted by the judicious choice of adjuvants. Various adjuvants with different fairly well-defined immunological activities (Allison, 1973; Frost and Lance, 1973; Dresser and Phillips, 1973; Johnson, 1974) were used, and their effects on the development of protection were monitored and compared. It was envisaged that these comparisons would provide direction for further studies.

\section{Material and methods}

\section{TEST ANIMALS}

New Zealand white rabbits were bred and raised indoors in the Medical Research Institute colony and fed Purina Rabbit Chow and water ad libitum. Rabbits in the MRI colony have never shown any physical evidence of infection with Treponema cuniculi or Rapid Plasma Reagin (RPR) antibodies (Small and Newman, 1972). The rabbits were 3 months old when vaccinated.

\section{ORGANISMS}

Treponema pallidum (Nichols pathogen) was acquired from infected rabbits sent from the Center for Disease Control, Atlanta, Georgia, April, 1972, and since passed intratesticularly.

\section{VACCINE PREPARATION}

Rabbits experimentally infected with $T$. pallidum showing a well-developed orchitis were killed by air embolism and their testes removed, minced, and dipped in PBS (pH 7.4) to remove loose cellular debris and extracellular tissue fluids. The testicular material was then swirled in fresh PBS for 30 to $60 \mathrm{~min}$. at $35^{\circ} \mathrm{C}$. The suspension of treponemes was centrifuged twice at $300 \mathrm{G}$ for 5 to $10 \mathrm{~min}$. and the number of treponemes counted in a PetroffHausser counting chamber under darkfield microscopy.
The $T$. pallidum whole cell suspensions that were free of whole cell testicular debris were ready for fixation. This was accomplished by adding glutaraldehyde to freshly harvested suspensions of unwashed spirochaetes for either $2 \mathrm{hrs}$ at room temperature or overnight at $4^{\circ} \mathrm{C}$., after which they were washed three times with PBS. Various concentrations of glutaraldehyde were tested to determine the weakest solution required to preserve the structural integrity of the spirochaete as seen by electron microscopy. An RR staining technique was performed on the treponenal suspensions of the vaccine preparation as reported by Zeigler and others (1976). This technique has determined the presence of an extracellular surface layer on treponemes.

\section{ADJUVANTS}

The following quantities of adjuvants were mixed with $3 \times 10^{8}$ glutaraldehyde-fixed $T$. pallidum (GFTP) per $0.5 \mathrm{ml}$. and injected into each of three rabbits:

(1) $1.5 \mathrm{ml}$. incomplete Freund's (IFA), (Difco Labs. Inc., Detroit, $\mathrm{Mi}$ );

(2) $1.5 \mathrm{ml}$. complete Freund's (CFA), (Difco);

(3) $100 \mathrm{mg}$. zymosan (ZYM), (Sigma Chemical Co., St. Louis, Mo);

(4) $0.5 \mathrm{mg}$. polyadenylic-polyuridylic acid (PAU), (Sigma);

(5) $0.5 \mathrm{ml}$. phytohaemagglutinin P (PHA), (Difco);

(6) $100 \mathrm{mg}$. alumina $C$ gel (ALC), (Sigma).

Five rabbits of the same age and sex were saved for challenge controls. All rabbits were vaccinated intraperitoneally (IP) except those receiving GFTP-PAU or GFTP alone which were vaccinated intravenously (IV).

\section{CHALLENGE}

5 months after vaccination, two animals of each vaccine group were challenged intratesticularly (IT) with $5 \times 10^{7}$ freshly harvested $T$. pallidum per testis. The remaining animal was challenged intradermally (ID) respectively with $0.1 \mathrm{ml}$. of $10^{7}, 10^{6}, 10^{5}, 10^{4}$, and $10^{3}$ freshly harvested $T$. pallidum cells on each side of its shaved back. This provided two inoculation sites per dilution tested. Also, two PBS controls were included on the back of each animal. IT and ID control animals were injected last. Challenge was evaluated by observation of testes or shaved backs for lesions. If a typical orchitis developed (or after $3 \mathrm{mths}$ ) the rabbit was killed by air embolism, the testes removed and sliced, and the testicular fluid examined for spirochaetes by darkfield microscopy. If intradermal lesions were seen, they were monitored later for induration, lanced, and examined by darkfield microscopy for the presence of treponemes.

\section{ANTIBODY RESPONSES}

Responses to cardiolipin antigen were monitored by the RPR card test* (Portnoy, Brewer, and Harris, 1962) while treponemal antibody was detected by the fluorescent treponemal antibody-absorbed (FTA-ABS) test (VDRL staff, 1968) using antigen and sorbent from Difco, and MRI-conjugated chicken antirabbit gamma globulin. Rabbit gamma globulin (RGG) was prepared according

^Hynson, Westcott and Dunning, Inc., Baltimore, Md 
to the method of Hebert, Pelham, and Pittman (1973). Protein content was determined by ultraviolet spectrophotemetry (Wells, Miller and Nadel, 1966).

Chickens were obtained from a local source, fed chicken mash and water ad libitum, and kept in separate cages. They were given Sulphaquinoxiline ${ }^{\star}$ in their drinking water for one week, rested for 3 weeks, and then injected with RGG. Injection dosage prescribed (Chase, 1967) was $40 \mathrm{mg}$. protein $/ \mathrm{kg}$. The chickens weighed about $1.5 \mathrm{~kg}$., so each received $60 \mathrm{mg}$. RGG in the wing vein on days $0,15,25$, and 37 , and were bled 2 and 6 days after the last injection. Sera were stored at $-20^{\circ} \mathrm{C}$.

Chicken antisera were fractionated three times with ammonium sulphate (Hebert, 1974), dialysed with saline and conjugated by the dialysis method (Clark and Shepard, 1963) to fluorescein isothiocyanate (FITC) $\dagger$ which was assayed greater than 99 per cent. pure by the Biological Stain Commission. F/P ratios were performed by spectrophotometric determinations at 493 and $278 \mathrm{~nm}$ (Wells and others, 1966). The FITC-conjugated chicken anti-RGG was tested according to standard methods (VDRL staff, 1968).

RPR and FTA-ABS antibodies were assayed in rabbit sera taken before vaccination, 3 and 17 weeks after vaccination, and 2 weeks after challenge.

\section{Results}

Fixation of T. pallidum cells

The minimum concentration of glutaraldehyde that preserved the integrity of the outer envelope of the treponemes as shown by electron microscopy was 1 per cent. (Fig. $1 a, b$ ). The 0.1 and 0.5 per cent. concentrations were unsuccessful in the time periods

*Vineland Poultry Labs., Vineland, N.J.

†International Biological Supplies, Melbourne, Fl tested and the 2 per cent. concentration was considered unnecessary. Thus, the 1 per cent. concentration of fixative was used for preparing the vaccine for this investigation. The RR electron microscopy studies by Zeigler and others (1976) showed the extracellular material well preserved with the use of 1 per cent. glutaraldehyde (Fig. 2a, $b$ ).

\section{Challenged-vaccinated rabbits}

Vaccinated rabbits were challenged with large doses of freshly isolated pathogenic $T$. pallidum, so that a lack of response would be more meaningful than if they had been challenged by a small dose. Thus, a comparative protection rating (CPR) could be established, reasonably based on differences in time of appearance of lesions and severity of infection in vaccinated rabbits compared to control rabbits.

Table I shows the results of the intradermally (ID) challenged rabbits and controls. Results were grouped separately as I and II because these rabbits were challenged on two different days. There are two results per challenge dose per rabbit because each was inoculated with the same dose on both sides of the mid-line of the back. Results are reported as the day the lesion first appeared.

In challenge group I, all rabbits formed ID lesions to challenge doses $10^{7}, 10^{6}, 10^{5}$, and $10^{4}$. However, only the GFTP vaccinated rabbit developed a lesion to the $10^{3}$ dose, developed ID lesions earlier than the control rabbit to the $10^{7}$ and $10^{4}$ doses, and had a larger number of indurated lesions. All three of these activities indicate an enhanced reaction to challenge.

TABLE I Lesion development in $\mathrm{T}$. pallidum vaccinated rabbits after intradermal challenge

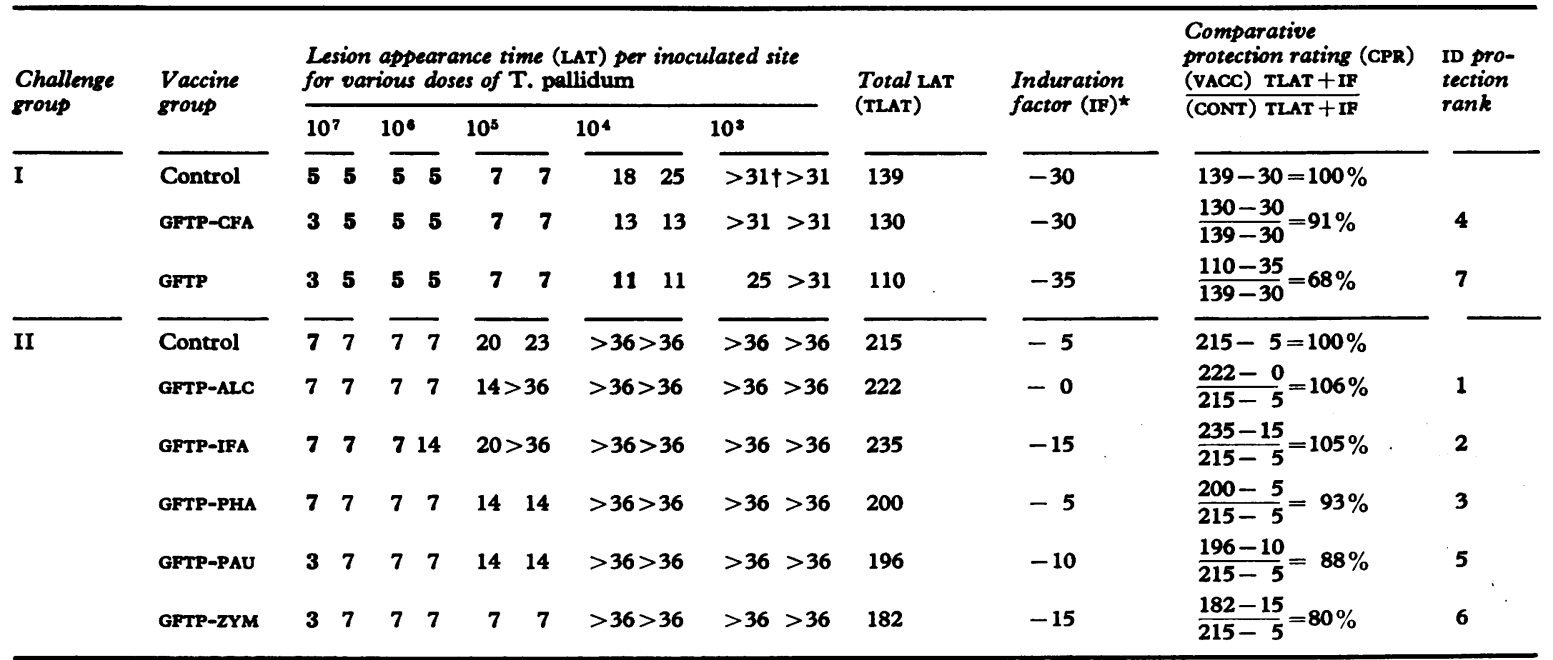

*Induration factor -5 points for each lesion which became indurated before rabbit was killed. Indurated lesions shown in bold type under LAT of inoculated site $(n)$.

tLesions had not appeared at these sites by the day the rabbits were killed. 
FIG. 1a Cross-section of T. pallidum cell harvested from infected rabbit testis, fixed with 1 per cent. glutaraldehyde, and then washed three times in PBS. Outer envelope $(O E)$, axial fibres $(A F)$, and inner envelope (IE) are present

FIG. 1b Cross-section of treponeme harvested from same infected rabbit testis as above but washed once in $P B S$ before fixation. Axial fibres $(A F)$ and inner envelope $(I E)$ are present but outer envelope is missing.

Bar equals $0.1 \mathrm{\mu m}$

In challenge group II, all rabbits developed ID lesions to the $10^{7}, 10^{8}$, and $10^{5}$ doses, but not to the $10^{4}$ and $10^{3}$ doses. Two vaccinated rabbits, GFTP-
ALC and GFTP-IFA, showed some protection by either greater time for lesion development, or fewer indurated lesions than the control rabbit. Rabbits 

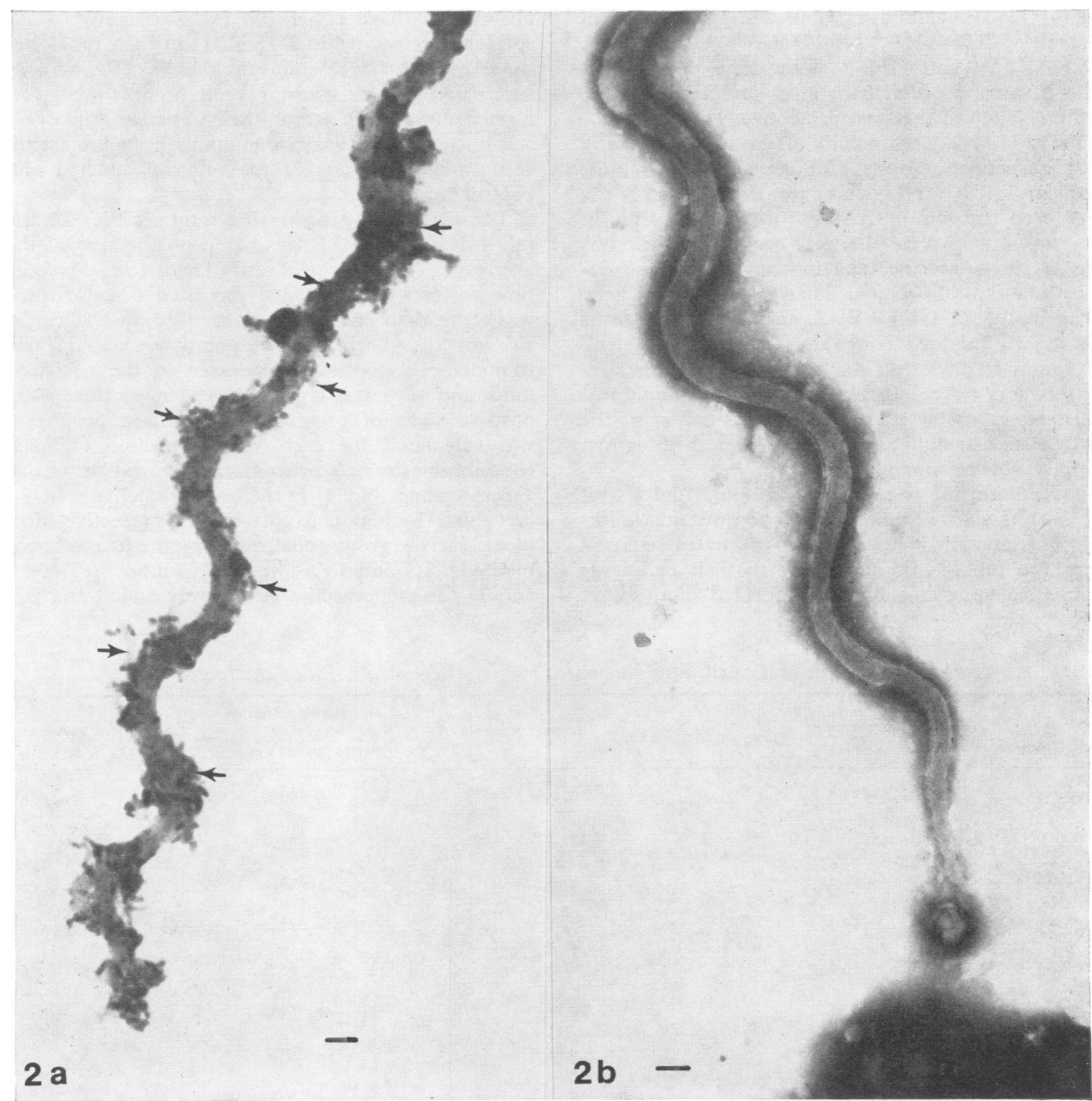

FIG. 2a Whole cell mount of treponeme harvested from infected rabbit testis, fixed with 1 per cent. glutaraldehyde, washed three times in PBS, and then reacted with $R R$. Note RR reaction product clumped at surface of cell (solid arrows)

FIG. 2b Whole cell mount of treponeme treated as above but not reacted with $R R$. Cell is negatively stained with ammonium molybdate. Note absence of clumped material at cell surface.

Bar equals 0.1 $\mathrm{um}$

vaccinated with GFTP-PHA, GFTP-PAU, and GFTP-ZYM showed negative protection by either less time for lesion appearance or greater number of indurated lesions.

To calculate a comparative protection rating (CPR) for the ID challenged rabbits, lesion appearance times were totalled (TLAT) for all inoculation sites in each rabbit. An arbitrarily-derived number of five points was subtracted for each indurated lesion; this was called the Induration Factor (IF) because it was a negative measure of protection. The sum of the Vaccine LAT and IF was divided by that of its control, and was designated the CPR. Table I reveals two rabbits with CPRs greater than control: GFTP-ALC 
and GFTP-IFA; and five rabbits with CPRs less than control: GFTP-PHA, GFTP-PAU, GFTP-CFA, GFTP-ZYM, and GFTP alone. The last column gives a ranking order from most protective vaccine group (No. 1) to the least protective (No. 7).

Table II shows the results of the intratesticularly (IT) challenged rabbits and controls. All rabbits received $5 \times 10^{7}$ treponemes per testis which is the dose used for routine passage of the organism. In this trial, in which rabbits were challenged 5 months after a single vaccine injection, all except one developed a typical orchitis. That exception had been vaccinated with GFTP-PHA and, while one testis was slightly enlarged 9 days after challenge, and small but lumpy 62 days after challenge, a typical large firm orchitis was never observed. When the rabbit was killed after 3 months, darkfield examination of the initial testicular fluid as well as a $30 \mathrm{~min}$. PBS extract failed to show a single spirochaete.

It is interesting to note that, although the rest of the rabbits showed a well-developed orchitis, darkfield examination of the fluid of the testes removed from the rabbits did not show that all had large numbers of spirochaetes as was expected. Testes were observed to have either few $(<5)$ or many $(>50)$ spirochaetes per field $(500 \times)$. Half of the rabbits in three of the GFTP-adjuvant (ALC, IFA, ZYM) vaccinated groups showed good orchitis with few numbers of spirochaetes in their testicular fluid. This was in contrast with the large number of spirochaetes seen in all members of the other vaccinated and control groups.

The comparative protecting rating (CPR) for the rabbits challenged IT was based on the sum of the average LATs and the severity factor (SF). Average time for lesion appearance was used because there was more than one rabbit in each group challenged IT. For SF, an arbitrary five points was added if few treponemes $(<5$ field) were seen in the testicular fluid, and 10 points if none was seen, as these were positive signs of protection. Percentage protection was calculated for each vaccine group. The last column gives a rank order from the most protective vaccine group (No. 1) to the least protective (No. 7).

Table III attempts to correlate the protective effect of the vaccine groups challenged by the two different methods, ID and IT. The CPRs found in Table I and II for each vaccine group were added and 200

TABLE II Lesion development in T. pallidum vaccinated rabbits after intratesticular challenge

\begin{tabular}{|c|c|c|c|c|c|c|}
\hline \multirow{2}{*}{$\frac{\text { Vaccine group }}{\text { Control }}$} & \multicolumn{2}{|c|}{$\frac{\text { Lesion appearance time }}{\text { No. of rabbits in group }}=$ Average LAT (a-LAT) } & \multirow{2}{*}{$\begin{array}{l}\text { Severity factor }(\mathrm{SF})^{\star} \\
0,0,0\end{array}$} & \multicolumn{2}{|c|}{ 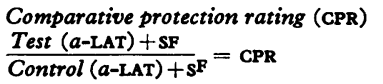 } & \multirow[t]{2}{*}{$\begin{array}{l}\text { IT } \\
\text { protection } \\
\text { rank }\end{array}$} \\
\hline & $\frac{41+14+15}{3}$ & $=23.3$ & & $\frac{23 \cdot 3+0}{23 \cdot 3}=100 \%$ & & \\
\hline GFTP-PHA & $\frac{86+14}{2}$ & $=50$ & 10,0 & $\frac{50+10}{23 \cdot 3}=261 \%$ & & 1 \\
\hline GFTP-ALC & $\frac{27+14}{2}$ & $=20 \cdot 5$ & 5,0 & $\frac{20 \cdot 5+5}{23 \cdot 3}=109 \%$ & & 2 \\
\hline GFTP-ZYM & $\frac{13+14}{2}$ & $=13.5$ & 0,0 & $\frac{13 \cdot 5+0}{23 \cdot 3}=58 \%$ & & 4 \\
\hline GFTP-CFA & $\frac{11+14}{2}$ & $=12 \cdot 5$ & 0,0 & $\frac{12 \cdot 5+0}{23 \cdot 3}=54 \%$ & & 5 \\
\hline GFTP & $\frac{11+11+7}{2}$ & $=9.6$ & $0,0,0$ & $\frac{9 \cdot 6+0}{23 \cdot 3}=41 \%$ & & 6 \\
\hline \multicolumn{7}{|c|}{$\begin{array}{l}\text { TABLE III Development of protection index }(P I) \text { from comparative protection ratings }(C P R) \text { of } I D \\
\text { and IT challenged rabbits }\end{array}$} \\
\hline \multicolumn{2}{|l|}{ Vaccine group } & \multicolumn{3}{|c|}{ Vacc. $(\mathrm{ID}+\mathrm{IT}$ CPR $\%)-$ Cont. $(\mathrm{ID}+\mathrm{IT}$ CPR $\%)=\mathrm{PI}$} & \multicolumn{2}{|l|}{ PI rank } \\
\hline $\begin{array}{l}\text { GFTP-PHA } \\
\text { GFTP-ALC } \\
\text { GPTP-IFA } \\
\text { GFTP-CFA } \\
\text { GFTP-ZYM } \\
\text { GFTP-PAU } \\
\text { GFTP }\end{array}$ & & $\begin{array}{l}(93 \%+261 \%)-(100 \%+ \\
(106 \%+109 \%)=(100 \%+ \\
(105 \%+82 \%)=(100 \%+ \\
(91 \%+54 \%)=(100 \%+ \\
(80 \%+58 \%)=(100 \%+ \\
(88 \%+39 \%)=(100 \%+ \\
(68 \%+41 \%)-(100 \%+\end{array}$ & $\begin{array}{l}p \%=154 \% \\
0 \%=15 \% \\
0 \%=-13 \% \\
0 \%=-55 \% \\
0 \%=-62 \% \\
0 \%)=-73 \% \\
0 \%)=-91 \%\end{array}$ & & $\begin{array}{l}1 \\
2 \\
3 \\
4 \\
5 \\
6 \\
7\end{array}$ & \\
\hline
\end{tabular}


per cent. (control per cent.) was subtracted from each total. The new sum was designated the Protection Index (PI). The vaccine groups with a positive PI were considered to have been partially protected, while those with a negative PI were considered to have had an enhanced infection. The last column gives a ranking order from most protective (No. 1) to least protective (No. 7) as follows: GFTP-PHA, GFTP-ALC, GFTP-IFA, GFTP-CFA, GFTPZYM, GFTP-PAU, and finally GFTP alone.

Rabbits vaccinated IV showed the least amount of protection. Thus the route of vaccination may be a factor in the development of protection or lack of it. Further studies of this factor are planned for the future.

\section{Antibody tests}

Table IV shows that 2 weeks after challenge (20 weeks after vaccination) rabbits injected IT showed stronger FTA-ABS and RPR antibody responses than those injected ID. However, the GFTP-CFA and GFTP vaccinates and the control group showed strong FTA-ABS and RPR antibody responses whether challenged ID or IT. The rabbit vaccinated with GFTP-PHA that never did form a typical orchitis after IT challenge, also had a weak FTAABS response. Rabbits with higher CPRs often showed weaker antibody responses especially after ID challenge. FTA-ABS and RPR assays of preliminary samples and 3 and 17 week post-vaccination samples were negative.

To counter a possible claim that poor infection and low FTA-ABS responses were caused by inoculating decreased numbers of virulent treponemes, all injections were made from pooled fresh $T$. pallidum harvests and control rabbits were injected last as a control for the interval between taking the treponemes out of the donor and injecting them into a new host.

\section{Discussion}

This study revealed that 1 per cent. glutaraldehyde fixation of pathogenic treponemes preserves the integrity of the outer envelope and also the extracellular material that has been clearly demonstrated to exist on the surface of $T$. pallidum cells by Zeigler and others (1976). By preserving both the outer envelope and the extracellular material, antigens have been included in this vaccine preparation that most likely have not been tested elsewhere, as other researchers (Metzger and others, 1969; Miller, 1973) prepared their vaccines with cells initially washed three times with saline.

Electron microscopy and RR staining techniques have been used to observe capsules on other bacteria (Chen, Elberg, Boyles, and Velez, 1975; Fletcher and Floodgate, 1973; Jones, Roth, and Sanders, 1969; Pate and Ordal, 1967; Springer and Roth, 1973). It has been reported (Luft, 1971) that $R R$ reacts with polyanions (chondroitin sulphate, hyaluronic acid, etc.) and phospholipids, suggesting that the extracellular material on treponemes may be polysaccharide in nature. Since polysaccharide antigens stimulate immune responses much less readily than do protein antigens, the treponemal extracellular coating may be responsible for the difficulty the host encounters producing humoral or cellular protective immune measures.

The data in this study reveal a measurable, differential effect of various treponemal-adjuvant vaccines upon the host's ability to provide resistance to a pathogenic challenge of $T$. pallidum 5 months after a single vaccine injection. Some vaccines

TABLE IV ID and IT antibody responses compared with ID and IT protection rankings from Tables $I$ and II

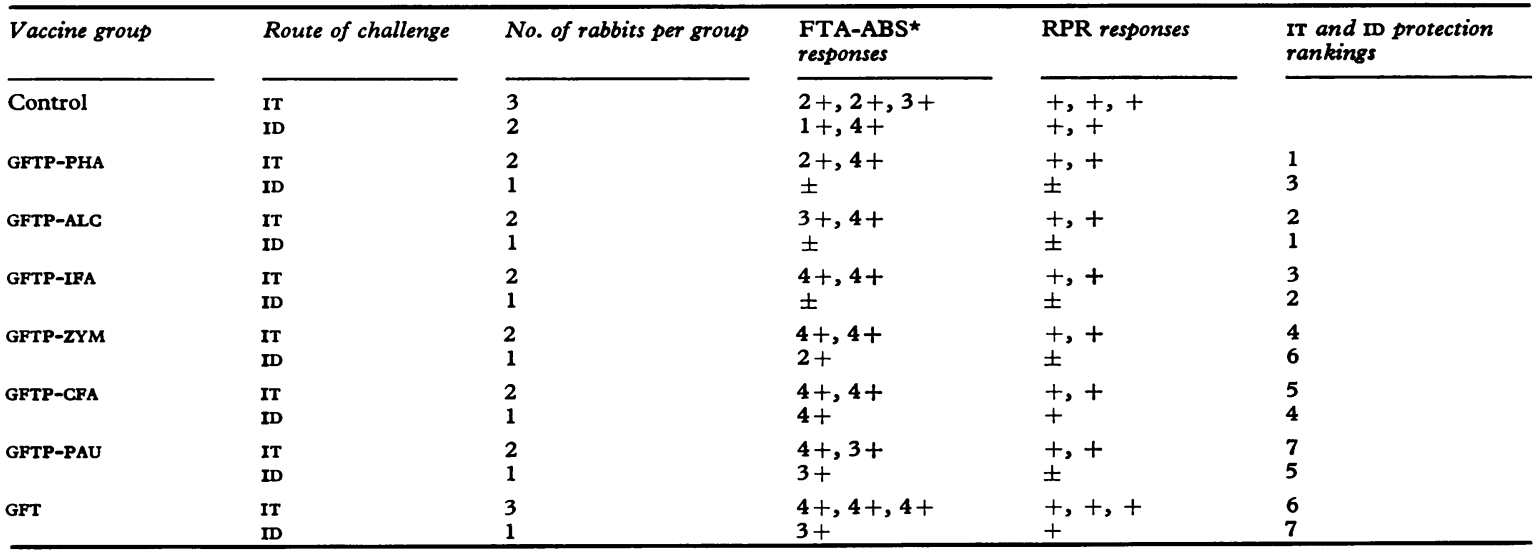

^FTA-ABS and RPR assays are from sera taken 2 weeks after challenge. FTA-ABS results are reported as negative $(-)$, borderline $( \pm)$, and reactive $(1$ to $4+$ ). RPR results are reported as negative $(-)$, weakly reactive $( \pm)$, or reactive $(+)$. 
stimulated partial protection, while others enhanced the infectivity of the challenge. This is demonstrated by the Protection Index (PI) which is based on lesion development in vaccinated rabbits compared to that in control rabbits. Positive PIs were observed for the GFTP-PHA and GFTP-ALC vaccines only. Although there were few animals tested in each vaccine group, the fact that the IT and ID rankings of both types of challenge were remarkably similar (Table IV) gives more credibility to the results.

Although the whole cell $T$. pallidum vaccine with its preserved outer envelope and slime layer was least effective in stimulating protection after only one injection compared to the vaccine preparations containing adjuvant, its ability to stimulate protection may be greater than the vaccines prepared by Metzger and others (1969) or Miller (1973), using their schedule of 27 and sixty injections respectively. However, more data are necessary to support such a statement. Since the use of a large number of vaccine injections is considered impracticable, effort in this laboratory has been devoted to the study of the use of a non-toxic adjuvant, effecting protection with a minimal number of vaccine injections.

Partial protection or infection enhancement was stimulated by adjuvants of which the effects on the immune mechanism are documented in several reviews (Allison, 1973; Dresser and Phillips, 1973; Frost and Lance, 1973; Johnson, 1974). Both T-cell (thymus-derived lymphocyte) stimulation and lymphocyte-trapping activities were helpful in establishing partial protection. These were functions of PHA and ALC adjuvants respectively.

The fact that protection was enhanced by $T$-cell and lymphocyte-trapping stimulators may indicate that these activities of the immune mechanism are suppressed during syphilitic infections. Musher, Schell, and Knox (1974) and Musher, Schell, Jones, and Jones (1975) have demonstrated that lymphocytes from the blood of persons with primary and secondary syphilis were less stimulated by treponemal antigens to undergo mitosis than lymphocytes from normal persons and cured syphilitics.

Another possible explanation for the enhanced protection shown in this study by PHA and ALC adjuvants is that the $T$. pallidum antigens alone are normally unable to initiate proper or effective $T$-cell activities or lymphocyte-trapping mechanisms. It is well known that certain antigens (most notably haptens) can stimulate primed cells for secondary responses, but need help (carrier) to initiate a primary response. While the adjuvant molecules in this study are probably not bound to the treponemal antigens covalently, the proper separate molecules may be sufficient to trigger the mechanisms of the lymphoidal cells to initiate protection.

Infection enhancement was greatest in those adjuvants that acted on macrophages (PAU and ZYM) and also on the development of delayed hypersensitivity (CFA). It is of interest that most of the researchers who tested adjuvants with syphilis vaccines used CFA (Magnuson and others, 1947); Eagle and Fleischman, 1948; Waring and Fleming, 1951; Izzat and Yazdi, 1973), and also found little enhancement of protection. Although delayed hypersensitivity reactions are known to require $T$-cell function, various subgroups of $T$-cells have been identified with different immunological activities (Amos, 1971; Federation Proceedings Abstracts, 1975).

Future work in this laboratory will be directed towards delineating the involvement of T-cells and B-cells and their subpopulations in experimental syphilis infection and protection by differential enhancement and differential inhibition of these populations in vivo.

This study was supported by PHS grants CC-00686 and CC-00687 from the Center for Disease Control, Atlanta, and grant 74346 from the John A. Hartford Foundation, New York City.

The authors are indebted to Kelly M. Mullin and W. Lee Blevins for their laboratory assistance.

\section{References}

Allison, A. C. (1973) In 'Ciba Foundation Symposium on Immunopotentiation', No. 18, p. 73. Ass. Sci. Publ., Amsterdam

Amos, B. (ed.) (1971) 'Progress in Immunology'. Chap. II. 'Lymphocyte Membranes'; Chap. V. 'Cell Cooperation in the Immune Response'; Chap. VI. 'Effector Mechanisms of Cell Mediated Immunity'. Academic Press, N.Y.

CHASE, M. W. (1967) 'Immunization of mammals other than man' in 'Methods in Immunology and Immunochemistry', ed. C. A. Williams and M. W. Chase, vol. 1, p. 230. Academic Press, New York

Chen, T. H., Elberg, S. S., Boyles, J., and Velez, M. A. (1975) Infect. and Immun., 11, 1382

Clark, H. F., and ShEPARD, C. C. (1963) Virology, 20, 642

Dresser, D. W., and Phillips, J. M. (1973) In 'Ciba Foundation Symposium on Immunopotentiation', No. 18, p. 3. Ass. Sci. Publ., Amsterdam

Eagle, H., and Fleischman, R. J. (1948) Amer. f. Syph., 87,369

Federation Proceedings Abstracts (1975) Cell Surface Markers, p. 945; Subpopulations of Immunologically Competent Cells, p. 986; T and B Lymphocytes and Cell Cooperation, p. 994. ed. P. L. Altman and D. S. Dittmer. Federation of American Societies for Experimental Biology, Bethesda, Md.

Fletcher, M., and Floodgate, G. D. (1973) f. gen. Microbiol., 74, 325

Frost, P., and LANCE, E. M. (1973) In 'Ciba Foundation Symposium on Immunopotentiation', No. 18, p. 29. Ass. Sci. Publ., Amsterdam

GlAUERT, A. M. (1975) 'Practical Methods in Electron Microscopy', p. 31. Elsevier Publ. Co., New York 
HeBert, G. A. (1974) Appl. Microbiol., 27, 389

-, Pelham, P. L., and Pittman, B. (1973) Ibid., 25, 26 IzZAT, N. N., KNOX, J. M., DACRES, W. G., and SMITH, E. B. (1971) Acta derm.-venereol. (Stockh.), 51, 157

- and YAZDI, E. (1973) Brit. F. vener. Dis., 49, 338

Johnson, A. G. (1974) 'Adjuvants to the Immune System' in Ann. Report in Med. Chem., ed. R. V. Henzelman, vol. 9, p. 244. Academic Press, New York JONES, H. C., ROTH, I. L., and SANDERS, W. M., III (1969) f. Bact., 99, 316

Jones, R. H., Nevin, T. A., Guest, W. J., and Logan, L. C. (1968) Brit. F. vener. Dis., 44, 193

LuFT, J. H. (1971) Anat. Rec., 171, 347

Magnuson, H. J., Halbert, S. P., and Rosenau, B. J. (1947) f. vener. Dis. Inform., 28, 267

Metzger, M., Michalska, E., PoDWiŃska, J., and SMOGóR, W. (1969) Brit. F. vener. Dis., 45, 299

- and PodwiŃska, J. (1967) Arch. Immunol. Ther. $\exp ., 15,34$
MILLER, J. N. (1973) F. Immunol., 110, 1206

MUSHER, D. M., SCHELL, R. F., JONES, R. H., and JONES, A. M. (1975) Infect. and Immun., 11, 1261

$\longrightarrow$, , and KNOX, J. M. (1974) Ibid., 9, 654

Pate, J. L., and Ordal, E. J. (1967) f. Cell Biol., 35, 37 Portnoy, J., Brewer, J. H., and Harris, A. (1962) Publ. Hlth Rep. (Wash.), 77, 645

Small, J. D., and Newman, B. (1972) Lab. Animal Sci., 22, 77

SPRINGER, E. L., and RoTH, I. L. (1973) F. gen. Microbiol., 74, 21

Venereal. Diskase Reskarch Laboratory StafF, CDC, Atlanta. (1968) Hlth Lab. Sci., 5, 23

WarING, G. W., Jr., and Fleming, W. L. (1951) Amer. $\mathcal{~ f . ~}$ Syph., 35, 568

Wells, A. F., Miller, C. E., and Nadei, M. K. (1966) Appl. Microbiol., 14, 271

ZeigleR, J. A., JoNes, R. H., Jones, A. M., and KubicA, K. M. (1976) Brit. F. vener. Dis., 52, 1 